\title{
Radiological pathogenesis of cervical myelopathy in 60 consecutive patients with cervical ossification of the posterior longitudinal ligament
}

\author{
Y Morio*,1, H Nagashima ${ }^{1}$, R Teshima ${ }^{1}$ and K Nawata ${ }^{1}$ \\ ${ }^{1}$ Department of Orthopaedic Surgery, Tottori University, Faculty of Medicine, Yonago, Japan
}

\begin{abstract}
Study design: The radiological pathogenetic factors for cervical myelopathy in 60 consecutive patients with cervical ossification of the posterior longitudinal ligament (OPLL) were investigated retrospectively.

Objective: To clarify which patients with OPLL will develop cervical myelopathy.

Methods: Sixty consecutive patients with OPLL were radiologically assessed comparing the myelopathic patient group (M group, $n=41$ ) and the mild or non-myelopathic patient group (non-M group, $n=19$ ).

Results: The narrowing ratio of the spinal canal in the $M$ group (47.1\%) was significantly greater $(P=0.026)$ than that in the non-M group $(38.3 \%)$. The two groups showed a significant difference $(P=0.0016)$ with regard to the Pavlov ratio (M group, 0.73 ; non-M group, 0.84$)$. The total range of motion of the cervical spine did not differ between the two groups but the per cent range of motion was significantly greater $(P=0.037)$ in the M group than in the non-M group.

Conclusion: This study suggests that factors important in the onset or aggravation of myelopathy are factors related to pathological compression by OPLL, cervical soft disc herniation, developmentally narrow spinal canal, and local or non-proportional hypermobility.
\end{abstract}

Keywords: cervical myelopathy; cervical ossification of the posterior longitudinal ligament

\section{Introduction}

Cervical ossification of the posterior longitudinal ligament (OPLL), as its size increases, is considered to compress the spinal cord or spinal nerve roots. ${ }^{1}$ Usually the clinical problems derive from the cervical myelopathy due to OPLL but radiologically apparent OPLL does not always induce clinical symptoms. Onset or aggravation of cervical myelopathy due to OPLL has been attributed to static compressive factors, such as the OPLL itself or soft disc herniation, ${ }^{2,3}$ and to dynamic factors of abnormal intervertebral movement, ${ }^{1,4-7}$ but it remains unclear which patients with OPLL will develop cervical myelopathy. This study investigated the radiological pathogenetic factors for cervical myelopathy in 60 consecutive patients with cervical OPLL retrospectively.

\section{Patients and methods}

The subjects were 60 patients with OPLL in the cervical spine, consisting of 37 men and 23 women aged 30-80 years (mean, 62.4 years). These patients consecutively consulted our clinic between January

*Correspondence: Y Morio, Department of Orthopaedic Surgery, Faculty of Medicine, Tottori University, 36-1 Nishimachi, Yonago, 683-8504, Japan
1990 and January 1997. Decompressive laminoplasty was performed in 30 of the 60 patients, anterior decompression with interbody fusion was performed in seven, while 23 patients were observed conservatively.

Magnetic resonance imaging (MRI) was performed in all patients before surgery or on the initial consultation with our clinic. A 1.0-T superconducting imaging system (Shimadzu SMT 100) or 1.5-T superconducting imaging system (Siemens Magnetome H15) was used for MRI. T1-weighted and T2weighted images of the cervical cord were obtained in the axial and sagittal views. Spinal cord signal intensity changes were evaluated. The disc level at which MRI revealed either intrinsic signal intensity change of the spinal cord or maximal compression of the spinal cord was considered the intervertebral disc level responsible for cervical myelopathy. The transverse area of the spinal cord at the site responsible for cervical myelopathy was computed on axial-view T1and $\mathrm{T} 2$-weighted images using a digitizer connected to a personal computer.

Lateral functional radiographs of the cervical spine were collected just before surgery or at the initial visit to our department. The intersectional angles at adjacent vertebrae in the cervical spine were measured, using a digitizer connected to a personal computer, on lateral radiographs for each patient in 
the neutral, maximal flexion and maximal extension positions. Each intersectional range of motion (ROM) was calculated as the difference between the intersectional angle at maximal flexion and that at maximal extension. ${ }^{8}$ The total ROM of the cervical spine was determined as the sum of the intersectional ROM from C1-2 to C6-7. The narrowing ratio of the spinal canal ${ }^{1}$ and the Pavlov ratio ${ }^{9}$ were measured on lateral radiographs in the neutral position (Figure 1). To assess dynamic factors for cervical myelopathy, we calculated the per cent ROM using the formula: per cent $\mathrm{ROM}=(\mathrm{ROM}$ at intervertebral disc level responsible for myelopathy/total $\mathrm{ROM}) \times 100$, or (ROM at intervertebral disc level of maximal compression of the spinal cord/total $\mathrm{ROM}) \times 100$.

The clinical grade of cervical myelopathy was evaluated according to the Japanese Orthopaedic Association score for cervical myelopathy (JOA score) immediately preoperatively or at the first consultation with our clinic and again at the final follow-up. The JOA score quantifies neurological impairment by evaluating upper extremity function (4 points), lower extremity function (4 points), sensitivity (6 points), and urinary bladder function (3 points). Therefore, a perfect JOA score is 17 points. Recovery after surgery was evaluated using the formula proposed by Hirabayashi: ${ }^{10}$ recovery rate $(\%)=(($ Postop. JOA Score - Preop. JOA Score $) /$ $(17-$ Preop. JOA Score $) \times 100$. The patients were divided into the myelopathic patient group (M

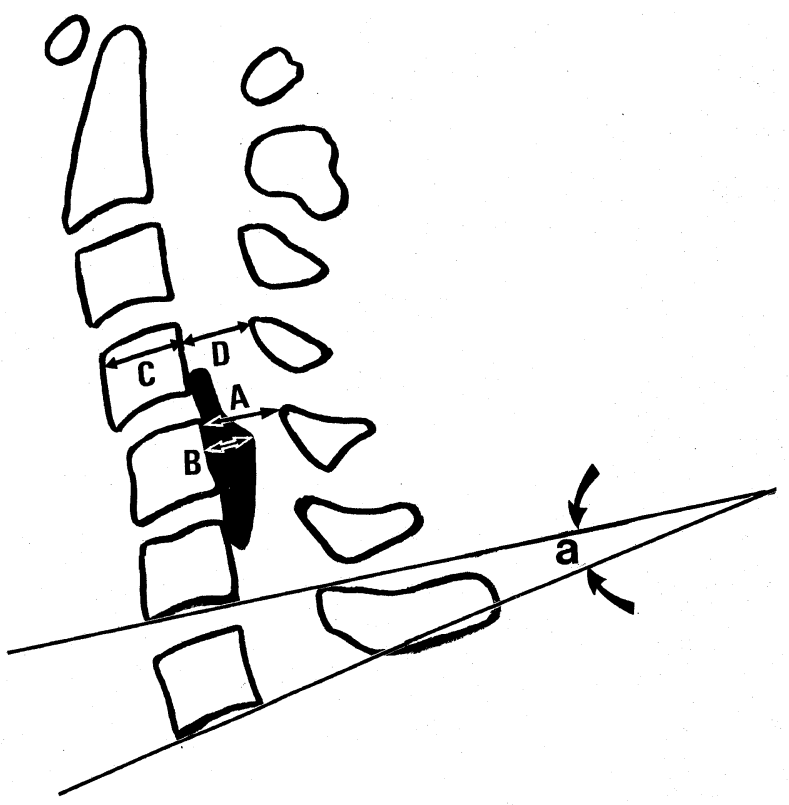

Figure 1 Measurement of the intersectional angle, the narrowing ratio of the spinal canal, and the Pavlov ratio. The narrowing ratio of the spinal canal $=\mathrm{B} / \mathrm{A} \times 100$, the Pavlov ratio $=\mathrm{D} / \mathrm{C}$, and the intersectional range of motion $=\mathrm{a}_{1}$ (at maximal extension) $-\mathrm{a}_{2}$ (at maximal flexion) group, $n=41$ ), in whom the JOA score was $4.5-14.5$ (mean, 10.3), and the mild or non-myelopathic patient group (non-M group, $n=19$ ), in whom the JOA score was 15-17 (mean, 16.8). Static factors (narrowing ratio and Pavlov ratio) and dynamic factors (total ROM and per cent ROM) were compared in the two groups.

The non-paired $t$-test was used for statistical comparisons of the two groups. Correlations between continuous data sets were tested with the Spearman's coefficient variants. A $P$ value of less than 0.05 was considered significant. Data are presented as mean values.

\section{Results}

Background features of the $M$-group and non- $M$ group The $M$ group consisted of 23 men and 18 women aged 37-80 years (mean, 60.9 years), while the non$\mathrm{M}$ group consisted of 14 men and five women aged 53-74 years (65.6 years). In the $M$ group, 29 patients with an average JOA score of 10.0 (4.513.0), underwent decompressive laminoplasty, seven patients with an average JOA score of 9.7 (5.0-13.0), underwent anterior decompression with interbody fusion, and five patients with an average JOA score of $12.9(9.5-14.5)$, received no surgical intervention due to other medical complications such as renal failure or refusal of surgery. Myelopathic symptoms in these patients without surgical intervention worsened during the course of conservative treatment. At the final follow-up (average 3 years after surgery), the average recovery rate in the laminoplasty patients and anterior interbody fusion patients were $48.5(0-100) \%$ and $51.9(20-100) \%$, respectively. In the non-M group, 18 patients received no surgery and one patient, with mild myelopathy (15 points) but severe intolerable paresthesia, underwent laminoplasty. The radiographical pattern of OPLL ${ }^{1}$ was mixed in 20 patients in the $\mathbf{M}$ group, segmental in 15 , and localized in six. The radiographical pattern of OPLL was continuous in five patients in the non$M$ group, mixed in six, segmental in six, and localized in two.

\section{$M R I$ assessment}

Four patterns of spinal cord signal intensity changes on T1-weighted sequences/on T2-weighted sequences were detected as follows: high signal intensity changes/high signal intensity changes (Hi/Hi); no change/high signal intensity changes (N/Hi); low signal intensity changes/high signal intensity changes $(\mathrm{Lo} / \mathrm{Hi})$; and no change/no change $(\mathrm{N} / \mathrm{N})$. In the $\mathrm{M}$ group, 36 patients showed $\mathrm{N} / \mathrm{Hi}$, two showed $\mathrm{Hi} / \mathrm{Hi}$, two showed $\mathrm{N} / \mathrm{N}$, and one showed $\mathrm{Lo} / \mathrm{Hi}$ signal pattern. The $\mathrm{N} / \mathrm{Hi}$ pattern at the site of cord compression was the most frequent MRI finding in this series of patients with OPLL and cervical 
myelopathy. In this group, cervical myelopathy was attributed to the OPLL itself in 17 patients in whom a signal intensity change of the spinal cord on MRI coincided with the maximally compressed segment (Figure 2) except in one patient with the $\mathrm{N} / \mathrm{N}$ pattern, to the dynamic factor in 17 patients in whom a signal intensity change on MRI did not coincide with the segment maximally compressed by OPLL except in one patient with the $\mathrm{N} / \mathrm{N}$ pattern, and to the soft disc herniation in seven patients (six with segmental OPLL type and one with mixed OPLL type) in whom a signal intensity change on MRI did not coincide with the segment maximally compressed by OPLL. OPLL itself induced cervical myelopathy in 17 patients, OPLL plus dynamic factors in 17 in whom the myelopathy-responsible level was discontinuous with the site of OPLL and at the caudal or cephalad edge of OPLL, and the soft disc herniation with OPLL in seven. In the non-M group there were 17 patients with $\mathrm{N} / \mathrm{N}$ and two with $\mathrm{N} / \mathrm{Hi}$ signal pattern.

The transverse area of the spinal cord at the myelopathy-responsible segment on T1-weighted and on T2-weighted images was $39.1 \pm 13.2 \mathrm{~mm}^{2}$ and $39.0 \pm 15.0 \mathrm{~mm}^{2}$, respectively, in the $\mathrm{M}$ group. The transverse area of the spinal cord at the site of maximal compression on T1-weighted and on T2- weighted images was $61.6 \pm 16.5 \mathrm{~mm}^{2}$ and $59.0 \pm 15.6 \mathrm{~mm}^{2}$, respectively, in the non-M group. There were significant differences between the groups with respect to the transverse spinal cord area at the site responsible for the cervical myelopathy or at the site of maximal compression (Table 1). There was a significant relationship between the JOA score and the transverse area of the spinal cord at the site responsible for the cervical myelopathy or at the site of maximal compression on T1-weighted images $\left(r_{\mathrm{s}}=0.588, P<0.0001\right)$ and on $\mathrm{T} 2$-weighted images $\left(r_{\mathrm{s}}=0.526, P=0.0002\right)$ as analyzed by the Spearman's coefficient variants.

\section{Radiological assessment}

The narrowing ratio of the spinal canal in the $M$ group was significantly greater than that in the non-M group. There was no significant relationship between the JOA score and the narrowing ratio of the spinal canal. The Pavlov ratio was 0.73 in the $\mathrm{M}$ group and 0.84 in the non-M group. The two groups showed a significant difference with regard to the Pavlov ratio. There was a significant relationship between the JOA score and the Pavlov ratio, as analyzed by the Spearman's coefficient variants $\left(r_{\mathrm{s}}=0.397\right.$, $P=0.0023)$. There was no significant difference
A

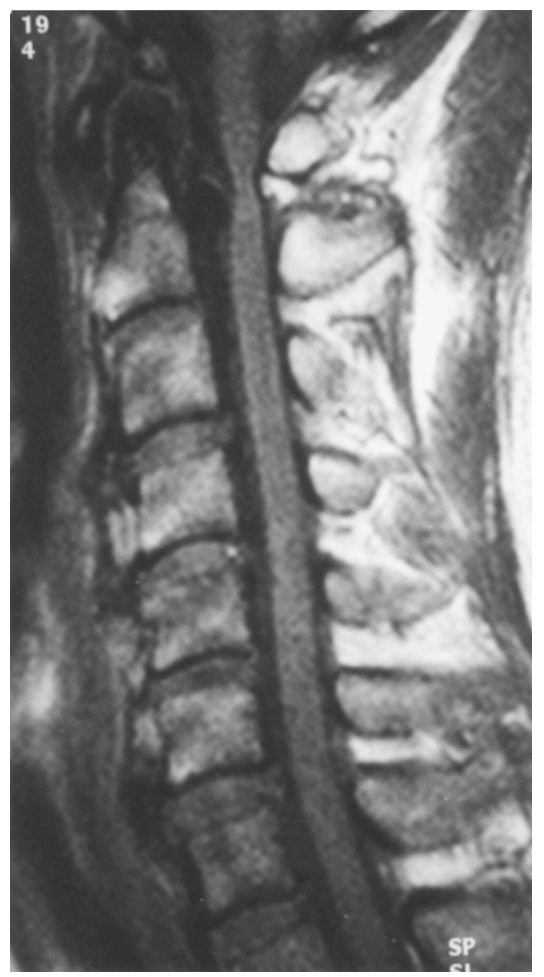

B

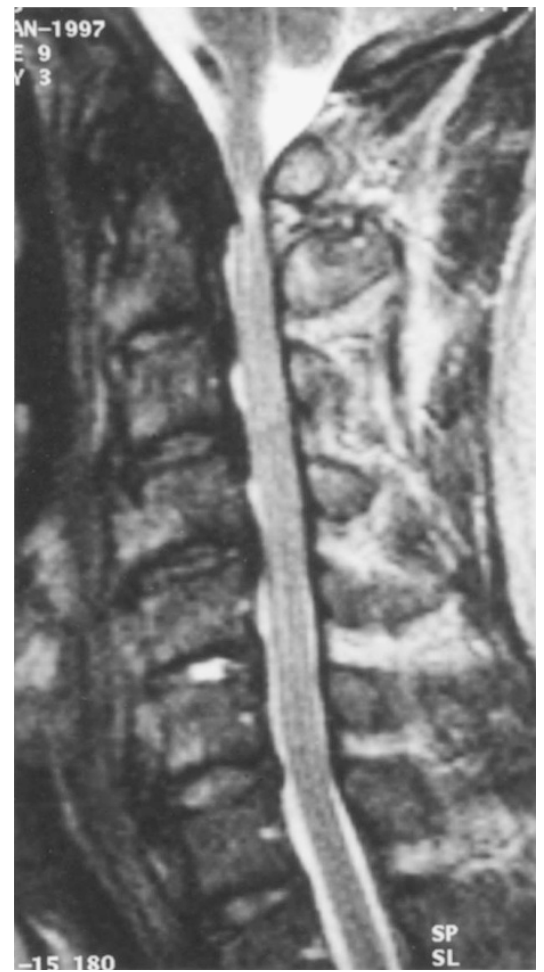

C

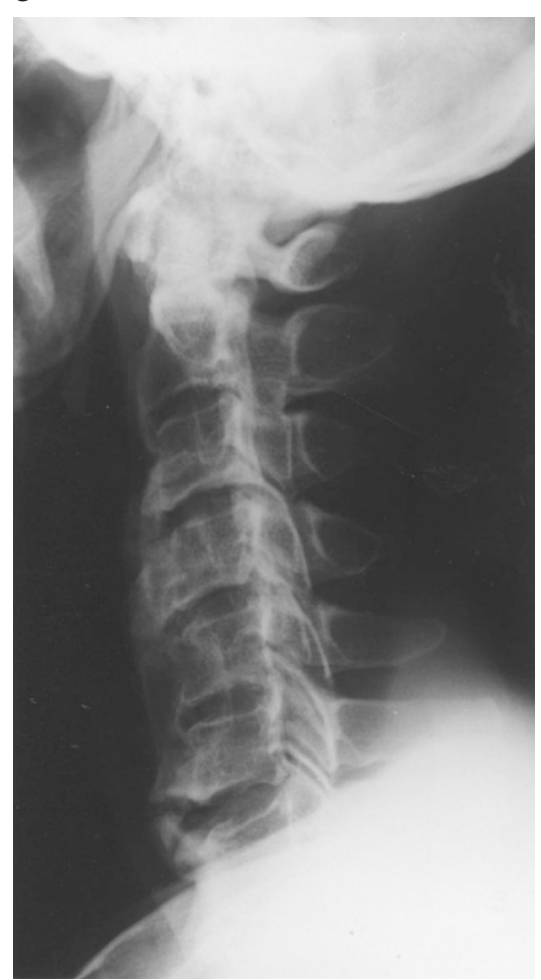

Figure 2 Radiograph and MR images for a 50-year old man with ossification of the posterior longitudinal ligament (C2-C6, mixed type). (A) T1-weighted image in sagittal view. (B) T2-weighted image in sagittal view. The compressed spinal cord at C1-2 did not show any signal intensity change on the T1-weighted image but high signal intensity change on the T2-weighted image. (C) Lateral X-ray view reveals narrowing ratio of $52.9 \%$, Pavlov ratio of 0.65 , and per cent $\mathrm{ROM}$ at $\mathrm{C} 1-2$ of $45.2 \%$ 
Table 1 Radiographic findings in 60 patients with cervical ossification of the posterior longitudinal ligament

\begin{tabular}{lccc}
\hline & $\begin{array}{c}\text { Patients with } \\
\text { myelopathy }(\mathrm{n}=41)\end{array}$ & $\begin{array}{c}\text { Patients without } \\
\text { myelopathy }(\mathrm{n}=19)\end{array}$ & \\
\hline Transverse area of the spinal cord at & & & \\
the site of maximal compression $\left(\mathrm{mm}^{2}\right)$ & & $61.6 \pm 16.5$ & $<0.0001 \#$ \\
T1-weighted images & $39.1 \pm 13.2$ & $59.0 \pm 15.6$ & $<0.0001 \#$ \\
T2-weighted images & $39.0 \pm 15.0$ & $38.3 \pm 11.3$ & $0.026 \#$ \\
Narrowing ratio $(\%)$ & $47.1 \pm 15.0$ & $0.84 \pm 0.12$ & $0.0016 \#$ \\
Pavlov ratio & $0.73 \pm 0.12$ & $46.5 \pm 15.2$ & $\mathrm{NS}$ \\
Total ROM $\left({ }^{\circ}\right)$ & $48.9 \pm 13.3$ & $15.4 \pm 10.1$ & $0.037 \#$ \\
Per cent ROM $(\%)$ & $20.7 \pm 8.5$ & & \\
\hline
\end{tabular}

\#: Student's non-paired $t$-test; NS: not significant

between the two groups with regard to the total ROM. The per cent ROM was $20.7 \%$ in the $\mathrm{M}$ group and $15.4 \%$ in the non-M group. There was a significant difference between the groups (Table 1). There were no relationships among the recovery rate and the Pavlov ratio, the narrowing ratio of the spinal canal, and the transverse area of the spinal cord at the site responsible for cervical myelopathy or at the site of the maximal compression on T1-weighted images and on T2-weighted images as analyzed by Spearman's coefficient variants.

\section{Discussion}

\section{Investigation method}

It has been reported that a change in the high signal intensity of the spinal cord on T2-weighted images reflects pathological changes in the spinal cord. ${ }^{11-13}$ In the current study, we presumed that the intervertebral disc level at which high signal intensity change of the spinal cord is recognized or at which the spinal cord is maximally compressed if it has no signal change, is responsible for myelopathic symptoms.

Use of the actual measurement in millimeters on lateral view of the cervical spine to document spinal stenosis is likely to be misleading because there is wide variance in the object-to-film distance and focus-to-film distance. ${ }^{9}$ For this reason we used the Pavlov ratio for assessing spinal canal stenosis. Matsunaga et $a l^{5}$ used a method of measuring the ROM in the cervical spine based solely on the relationship between the $\mathrm{C} 1$ and $\mathrm{C} 7$ vertebrae. We emphasize that each intersectional ROM is essential since the spinal cord is usually damaged at one or two intervertebral disc levels in OPLL patients, and the ROM of the cervical spine based on the sum for all intersectional ROM more suitably reflects the pathological condition of OPLL than C1-C7 ROM and the per cent ROM, which does not mean absolute values of the local ROM but the comparative rates of the local ROM, is a novel parameter reflecting local and non-proportional hypermobility.
Pathogenesis

Despite many reviews ${ }^{14,15}$ of the mechanism of cervical spondylotic myelopathy, there are few reports as to the mechanism of spinal cord damage by OPLL. ${ }^{16}$ Concerning the causes of myelopathy in these patients with cervical OPLL, the present results suggest that the compressive factors of OPLL and the developmental spinal canal stenosis are both static factors and that both are important in the onset or aggravation of myelopathic symptoms. In addition, we also consider soft disc herniation to be another static compressive factor. Hanakita et $a l^{2}$ and Kokubun et $a l^{3}$ also focused attention on the cervical soft disc herniation which induced myelopathic symptoms in patients with radiologically apparent OPLL of the segmental type.

Kukita et $a l^{4}$ and Matsunaga et $a l^{5}$ reported limited total ROM of the cervical spine in some patients without myelopathic signs, despite severe spinal stenosis. Our study, in contrast, did not disclose any significant relationship between the total ROM of the cervical spine and presence of the myelopathic symptoms. However, the per cent ROM at each disc level responsible for myelopathy in the $\mathrm{M}$ group was greater than that in the non-M group. These results suggest that local and non-proportional slight movement of the mobile segment at the myelopathy-responsible intervertebral disc level could add critical insult to the spinal cord compressed by slowly enlarging OPLL. To our knowledge, there are no reports demonstrating that local and nonproportional hypermobility of the mobile segment could add critical damage to the spinal cord compressed by slowly increasing OPLL. This local and non-proportional hypermobility is very important because this information could be used to develop a new treatment strategy. There are already two options of anterior decompression with fusion and posterior decompression. We can now add another surgical option of anterior interbody fusion without decompression for the local or non-proportional hypermobile segment.

In conclusion, the factors important in the onset or aggravation of myelopathy are factors related to pathological compression by OPLL, those related to 
pathological compression by cervical soft disc herniation, developmentally narrow spinal canal, and local or non-proportional hypermobility.

\section{References}

1 Tsuyama N. Ossification of the posterior longitudinal ligament of the spine. Clin Orthop 1984; 184: $71-84$.

2 Hanakita $\mathrm{J}$ et al. The significance of the cervical soft disc herniation in the ossification of the posterior longitudinal ligament. Spine 1994; 19: 412-418.

3 Kokubun S, Nishihira T. Relationship of disc herniation in OPLL of the cervical spine. Investigation Committee Report on OPLL from Japanese Ministry of Public Health and Welfare, $1984 ; 179-182$

4 Kukita $\mathrm{M}$ et al. Clinical course and mechanism of myelopathy on ossification of the posterior longitudinal ligament in the cervical spine. Rinsho Seikei Geka 1998; 33: 419-423.

5 Matsunaga $\mathrm{S}$ et al. The natural course of myelopathy caused by ossification of the posterior longitudinal ligament in the cervical spine. Clin Orthop 1994; 305: 168 - 177.

6 Seki $\mathrm{H}$ et al. Clinical evaluation of 185 cases with cervical ossification of the posterior longitudinal ligament. Seikeigeka (Orthopedic Surgery) 1974; 25: 704-710.

7 Tominaga S. Conservative treatment for stenotic-compressive myelopathy due to OPLL. Seikeigeka (Orthopedic Surgery) 1993; 44: $1189-1196$.
8 Inoue A, Ikata T, Katoh S. Spinal deformity following surgery for spinal cord tumors and tumorous lesions: analysis based on an assessment of the spinal functional curve. Spinal Cord 1996; 34: $536-542$

9 Pavlov H, Torg JS, Robie B, Jahre C. Cervical spinal stenosis: determination with vertebral body ratio method. Radiology 1987; 164: $771-775$.

10 Hirabayashi $\mathrm{K}$ et al. Operative results and postoperative progression of ossification among patients with ossification of cervical posterior longitudinal ligament. Spine 1981; 6: 354-364.

11 Takahashi M, Yamashita T, Sakamoto Y, Kojima R. Chronic cervical cord compression: clinical significance of increased signal intensity of MR images. Radiology 1989; 173: 219-224.

12 Ramanauskas WL et al. MR imaging of compressive myelopathy. J Comput Assist Tomogr 1989; 13: 399-404.

13 Al-Mefty $\mathrm{O}$ et al. Myelopathic cervical spondylotic lesions demonstrated by magnetic resonance imaging. J Neurosurg 1988; 68: $217-222$.

14 Krauss WE, McCormick PC. Cervical spondylotic myelopathy. Semin Neurol 1993; 13: $343-348$.

15 Law MD, Bernhardt M, White AA. Evaluation and management of cervical spondylotic myelopathy. J Bone Joint Surg [Am] 1994; 76: $1420-1433$.

16 Hashizume Y et al. Pathology of spinal cord lesions caused by ossification of the posterior longitudinal ligament. In: Yonenobu $\mathrm{K}$, Sakou T, Ono K (ed). OPLL: ossification of the posterior longitudinal ligament. Springer-Verlag, Tokyo, 1997, pp 59-64. 